\section{Enfermedad celíaca silente en epilepsia criptogénica del adulto}

\author{
ANA MARÍA MADRID S. ${ }^{1}$, MARIO DÍAZ S. ${ }^{2}$, CARMEN HURTADO H. ${ }^{1, \mathrm{a}}$, \\ LEDDA AGUILERA O. ${ }^{2}$, BORIS MENA U. ${ }^{2}$
}

\section{Silent celiac disease among 21 patients with cryptogenic epilepsy}

Background: Celiac disease (CD) is predominant in women and young people. Atypical, non-enteric symptoms are more common among adults. There is also an association between $C D$ and neurological disorders, especially with cerebellar ataxia, polyneuropathy and epilepsy. Aim: To study the frequency of CD in a group of adults with cryptogenic epilepsy. Material and Methods: Twenty one patients with cryptogenic epilepsy, aged 20 to 65 years (14 women) were studied, measuring IgA-anti transglutaminase antibodies and deamidated gliadin peptide (DGP) IgG and IgA antibodies. Results: One patient had elevated titers of both types of antibodies. Small bowel biopsy showed villous atrophy and lymphocytic infiltration compatible with CD. Conclusions: One of 21 adult patients with cryptogenic epilepsy had a silent $C D$.

(Rev Med Chile 2011; 139: 587-591).

Key words: Celiac disease; Epilepsy; Gliadin; Plasma transglutaminase.

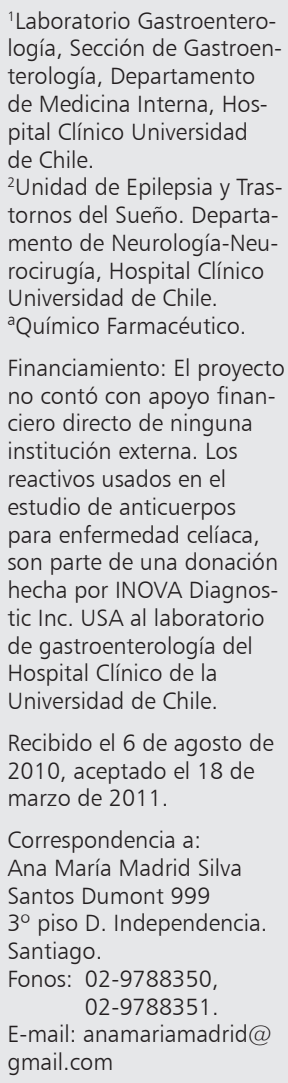

L a enfermedad celíaca (EC) es una enteropatía inflamatoria provocada por una reacción autoinmune a la proteína del gluten que afecta a individuos genéticamente susceptibles. La prevalencia de la EC en distintos países varía entre el $0,3 \%$ y $1 \%{ }^{1}$. La enfermedad afecta más a personas jóvenes, especialmente mujeres, siendo la presentación clínica habitual diversos síntomas gastrointestinales asociados a un síndrome de mala absorción. Sin embargo, en pacientes de mayor edad predominan las formas atípicas que se presentan asociadas a diversos trastornos extraintestinales ${ }^{2,3}$, dentro de los que destacan afecciones neurológicas como ataxia cerebelosa, polineuropatía, leucoencefalopatía y epilepsia ${ }^{4-6}$. En estos casos el diagnóstico de EC se confirma con estudios inmunológicos y biopsia de intestino delgado.

Los mecanismos fisiopatológicos de las alteraciones neurológicas en la EC no están bien esclarecidos, atribuyéndolos a la generación de anticuerpos antineuronales ${ }^{7}$. En las últimas dos décadas se han publicado numerosas investigaciones que dan cuenta de una mayor prevalencia de EC silente en pacientes con epilepsia. En 1992 Gobbi fue el primero en demostrar una asociación directa entre la EC y epilepsia con calcificaciones occipitales $^{8}$.

Nuestro trabajo tuvo como objetivo estudiar la prevalencia de EC en un grupo de pacientes adultos con epilepsia criptogénica a través de la medición de anticuerpos específicos con dos técnicas. Se discute además la evidencia actual de asociación entre ambas patologías.

\section{Material y Métodos}

Los pacientes ingresados al estudio provienen del Policlínico de Epilepsias del Hospital Clínico de la Universidad de Chile que consultaron entre enero y diciembre de 2008. Este policlínico especializado generalmente atiende pacientes de difícil manejo. 
Se seleccionaron aquellos pacientes controles y nuevos portadores de un síndrome epiléptico criptogénico, es decir, aquellos casos en los que además de haberse descartado síndromes claramente hereditarios no se ha podido demostrar la etiología de la enfermedad a través de distintos estudios. Los pacientes de estas características corresponden aproximadamente al 10\% del total de las consultas.

Los criterios de inclusión fueron: edad entre 18 y 65 años, epilepsia de al menos un año de evolución al momento de la consulta, estar o haber estado en tratamiento con algún fármaco antiepiléptico (FAE), resonancia magnética cerebral con protocolo para epilepsia normal.

Los criterios de exclusión fueron: pacientes con antecedentes previos de EC o alguna otra enteropatía. Diagnóstico previo de enfermedad autoinmune o enfermedad neurológica progresiva, casos en los que se sospechen o hayan sido confirmadas pseudocrisis psicógenas, epilepsia sintomática o síndromes generalizados idiopáticos bien reconocidos como hereditarios (ej: epilepsia mioclónica juvenil).

En cada uno de los pacientes se obtuvo sus antecedentes clínicos y se extrajo una muestra de sangre para estudio de anticuerpos específicos de EC. Cada muestra se analizó mediante técnica de ELISA semicuantitativo (Quanta Lite ${ }^{\circledR}$, INOVA Diagnostics ${ }^{\circledR}$ ) para determinación de anticuerpos antitransglutaminasa tisular IgA (ATG-A), utilizada de rutina en nuestro laboratorio y la nueva técnica de "celiac DPG screen" (DPG), la cual puede detectar simultáneamente anticuerpos del tipo IgG e IgA contra péptidos deaminados de gliadina. Esta última técnica presenta alta sensibilidad y especificidad y se usó como complemento para el caso que existan pacientes deficientes de $\operatorname{IgA}^{9,10}$. En ambas técnicas los resultados se consideraron negativos con cifras menores a 20 UI.

A todos los pacientes se les explicó los objetivos del estudio y firmaron un consentimiento informado. La investigación fue aprobada por el Comité de Ética del Hospital Clínico de la Universidad de Chile.

\section{Resultados}

Se incluyeron 21 pacientes que cumplían con los criterios del estudio (Tabla 1 ). La edad promedio de la muestra fue de 41,3 años (20-65 años), 7 hombres $(33,3 \%)$ y 14 mujeres $(66,7 \%)$. La edad de inicio promedio de la epilepsia fue de 29,3 años (rango: 6-62 años de edad). La duración de la enfermedad fluctuaba entre 1 y 59 años.

La gran mayoría de los casos correspondían a síndromes epilépticos focales (17/21 pacientes). El rango de frecuencia de crisis fue muy variable caso a caso (1-204 eventos/año), existiendo un paciente que se encontraba libre de crisis en los últimos 5 años de control.

Nueve pacientes $(42 \%)$ se mantenían en tratamiento con al menos 2 FAE, 11 (52\%) estaban en monoterapia y 1 paciente no había recibido fármacos en los 2 últimos años de enfermedad. Las alteraciones del EEG fueron variables, incluyendo casos con actividad lenta focal, actividad epileptiforme focal o generalizada. Tres pacientes $(14,2 \%)$ tenían EEGs normales.

Se observó una alta concordancia entre las dos técnicas para el diagnóstico de EC (Tabla 1). Del total de casos se encontró sólo un paciente de sexo femenino con títulos positivos elevados en ambas técnicas (4,7\%). La paciente, de 33 años de edad, fue derivada a nuestra unidad desde la ciudad de Ovalle con historia de crisis tónico clónicas generalizadas y crisis parciales complejas de 13 años de evolución. Tenía una baja frecuencia de las crisis descritas ( \pm 2 crisis por año), sin una adecuada respuesta al uso prolongado de fenitoína y ácido valproico. Durante los últimos 3 años se agregaron a las crisis habituales algunas que se iniciaban con alucinaciones visuales caracterizadas por la aparición repentina de figuras en el hemicampo visual derecho que la paciente describía como "comics" y que posteriormente evolucionaban hacia crisis parciales complejas. La RM cerebral con protocolo para epilepsia fue normal. Tenía varios EEG normales y uno que describía una discreta lentitud intermitente temporal izquierda de carácter inespecífica. El estudio bioquímico e inmunológico en sangre fue normal. Se le indicó cambiar el tratamiento a carbamazepina y se le invitó a participar en este estudio, encontrándose títulos elevados de ATG-A y DPG en sangre (47 y 40 UI respectivamente). No refería antecedentes de diarrea, anemia y dolor abdominal. Al interrogatorio dirigido sólo aquejaba distensión abdominal. Se le indicó realizar una endoscopia digestiva alta con biopsia duodenal. Se tomaron 12 muestras en forma escalonada desde la cuarta porción del duodeno hasta el bulbo, cuyo estudio histológico 
Enfermedad celíaca en epilepsia criptogénica - A. M. Madrid et al

Tabla 1. Características clínicas de 21 pacientes con epilepsia criptogénica con estudio de anticuerpos anti-transglutaminasa-A y DPG screen

\begin{tabular}{|c|c|c|c|c|c|c|}
\hline Caso & $\begin{array}{l}\text { Años de } \\
\text { epilepsia }\end{array}$ & $\begin{array}{c}\text { Nivel } \\
\text { anti-TGA } \\
\text { (UI) }\end{array}$ & $\begin{array}{l}\text { Nivel } \\
\text { DGP screen } \\
\text { (UI) }\end{array}$ & Crisis/año & Tipos de crisis & $\begin{array}{l}\text { Fármacos } \\
\text { antiepilépticos }\end{array}$ \\
\hline 1 & 1 & 4 & 5 & 12 & TCG & OXC, PDN \\
\hline 2 & 3 & 9 & 7 & 1 & TCG & AV \\
\hline 3 & 9 & 6 & 4 & 20 & PS MOTORA, TCG & OXC, LTG, GCB \\
\hline 4 & 1 & 4 & 11 & 1 & TCG & NO \\
\hline 5 & 12 & 3 & 3 & 12 & CPC, TCG & AV \\
\hline 6 & 13 & $47 *$ & $40 *$ & 2 & PS VISUAL, CPC, TCG & CBZP, CLOB \\
\hline 7 & 25 & 5 & 5 & 0 & PS, TCG & CBZP \\
\hline 8 & 59 & 11 & 11 & 48 & AUSENCIAS, TCG & AV \\
\hline 9 & 7 & 6 & 4 & 24 & PS MNESICA, TCG & CZP \\
\hline 10 & 3 & 10 & 8 & 2 & CPC, PS MNESICA & OXC, FBB \\
\hline 11 & 2 & 3 & 3 & 1 & TCG, STATUS CONVULSIVO & LTG \\
\hline 12 & 32 & 6 & 5 & 204 & CPC, MIOCLONIAS, TCG & AV, TPM, CLOB \\
\hline 13 & 7 & 8 & 8 & 2 & PS MOTORA, TCG & OXC \\
\hline 14 & 10 & 4 & 4 & 0 & CPC, TCG & CBZP \\
\hline 15 & 6 & 8 & 7 & 4 & TCG & LEV \\
\hline 16 & 23 & 4 & 4 & 8 & CPC, TCG & OXC, LTG, AV \\
\hline 17 & 4 & 4 & 5 & 3 & PS MOTORA, TCG & LEV \\
\hline 18 & 11 & 8 & 4 & 2 & CPC, PS MOTORA, TCG & FNT \\
\hline 19 & 14 & 4 & 5 & 72 & CPC, TCG & LEV, AV, CLOB \\
\hline 20 & 6 & 5 & 5 & 0 & TCG & CBZP \\
\hline 21 & 3 & 6 & 4 & 24 & PS VISUAL, CPC & CBZP, FBB \\
\hline
\end{tabular}

*caso descrito de paciente con EC y epilepsia criptogénica. (TCG: tónico-clónico-generalizada, PS: parcial simple, CPC: crisis parcial compleja). (CBZP: carbamazepina, AV: ácido valproico, CLOB: clobazam, LTG: lamotrigina, OXC: oxcarbazepina, FBB: fenobarbital, LEV: levetiracetam, PDN: primidona).

mostró un aspecto atrófico de la mucosa con infiltrado linfocitario, hiperplasia de criptas y atrofia vellositaria, compatibles con el diagnóstico de EC Marsh IIIb.

Se inició terapia con restricción de gluten en marzo de 2008, manteniéndose sin crisis epilépticas hasta la fecha. En el último control médico (marzo 2010), se evidenció normalización de los títulos de ATG-A.

\section{Discusión}

En nuestro trabajo se encontró que 1 de 21 pacientes $(4,7 \%)$ adultos con epilepsia criptogénica era portador de una EC silente, considerando la ausencia de síntomas gastrointestinales. El diagnóstico se basó en la presencia de niveles altos de ATG-A y DPG en sangre y la posterior confirmación histológica con biopsia duodenal. En marzo de 2008 se inicia terapia con restricción de gluten, manteniéndose sin ningún tipo de crisis hasta el último control en marzo 2010, lo que podría atribuirse tanto a la dieta como a la introducción de carbamazepina en su terapia, destacando el hecho que se normalizaron los niveles de ATG-A.

Pese a que se estudió una cohorte pequeña de pacientes, no tenemos referencias de otros estudios similares en poblaciones seleccionadas de 
epilépticos. A diferencia de otras publicaciones, no incluimos en el estudio la medición de anticuerpos antigliadina debido a que comparativamente presentan una menor sensibilidad y especificidad con las técnicas por nosotros utilizadas para el diagnóstico de EC, y sólo indican sensibilidad a la proteína del gluten, pudiendo incluso estar elevados hasta en $10 \%$ de la población sana. Los ATG-A tienen una alta especificidad y sensibilidad para el diagnóstico de EC siendo la herramienta más ampliamente utilizada, pero debido a que mide sólo anticuerpos IgA, se utilizó la técnica DPG que mide simultáneamente anticuerpos IgG e IgA contra péptidos desaminados de gliadina y que ha demostrado tener una mayor sensibilidad y especificidad.

Aparentemente el síndrome descrito por Gobbi que asocia epilepsia, calcificaciones occipitales y EC se encuentra completo sólo en un grupo de pacientes, la mayoría de ellos de origen italiano. El mismo autor publicó posteriormente una extensa revisión en la que se incluían pacientes sin calcificaciones cerebrales ${ }^{9}$. También se han publicado varios estudios poblacionales que han mostrado una mayor prevalencia de EC silente en pacientes epilépticos respecto a controles sanos ${ }^{10,11}$, sin que exista una clara asociación con calcificaciones cerebrales. Se postula que factores ambientales o genéticos en la población inicialmente descrita por Gobbi serían los responsables de la aparición de calcificaciones cerebrales; de hecho se han descrito casos en Argentina, donde se sabe existe un alto número de inmigrantes italianos y en países mediterráneos como España ${ }^{12}$.

También se han publicado trabajos que muestran una débil o nula asociación entre epilepsia y EC ${ }^{13}$. Ranua et al, al estudiar 968 pacientes v/s controles sólo encontraron una mayor prevalencia de anticuerpos antigliadina elevados en pacientes con síndromes epilépticos primariamente generalizados ${ }^{14}$.

La respuesta al tratamiento con restricción de gluten es variable, describiéndose casos en los que disminuye notoriamente el número de crisis epilépticas ${ }^{15-17}$. En Chile, previamente se publicó el caso de una paciente adulta con una epilepsia refractaria a quien se le diagnosticó EC por estudio inmunológico y biopsia de intestino delgado, la frecuencia de crisis bajó notoriamente al indicársele una dieta libre de gluten ${ }^{18}$.

No están claros los mecanismos por los cuales ambas patologías estén asociadas, aunque la teoría más aceptada es que en algunos pacientes con EC se producirían anticuerpos antineuronales responsables de la aparición de cuadros neurológicos como ataxia y epilepsia.

En nuestra serie se observó una buena concordancia entre ambas técnicas diagnósticas de EC. Ninguna de las muestras negativas para ATG-A resultó positiva para DPG.

Finalmente, creemos que nuestros resultados justifican la realización futura de estudios con poblaciones más numerosas de epilépticos, incluyendo un grupo control con epilépticos sintomáticos bien definidos, para determinar la real prevalencia de la EC en casos en los que la etiología de la epilepsia no está clara. De confirmarse una asociación, se debería incluir un estudio de EC silente en pacientes con epilepsia de etiología no aclarada.

\section{Referencias}

1. Collin P. Should adults be screened for celiac disease? What are the benefits and harms of screening? Gastroenterology 2005; 128 (4 Suppl 1): S104-8.

2. Canales P, Araya M, Alliende F, Hunter B, Alarcón T, Chávez E. Estado actual del diagnóstico y presentaciones clínicas de enfermedad celíaca. Estudio multicéntrico. Rev Med Chile 2008; 136: 296-303.

3. Heredia C, Castro F, Palma J. Enfermedad Celíaca del adulto. Rev Med Chile 2007; 135: 1186-94.

4. Zelnik N, Pacht A, Obeid R, Lerner A. Range of neurologic disorders in patients with celiac disease. Pediatrics 2004;113: 1672-6.

5. Pengiran Tengah DS, Wills AJ, Holmes GK. Neurological complications of coeliac disease. Postgrad Med J 2002; 78 (921): 393-8.

6. Bushara KO. Neurologic presentation of celiac disease. Gastroenterology 2005; 128 (4 Suppl 1): S92-7.

7. Briani C, Zara G, Alaedini A, Grassivaro F, Ruggero S, Toffanin E, et al Neurological complications of celiac disease and autoimmune mechanisms: a prospective study. J Neuroinmunology 2008; 195: 171-5

8. Gobbi G, Bouquet F, Greco L, Lambertini A, Tassinari CA, Ventura A, et al. Coeliac disease, epilepsy, and cerebral calcifications. Lancet 1992; 22; 340 (8817): 439-43.

9. Gobbi G. Coeliac disease, epilepsy and cerebral calcifications. Brain Dev 2005; 27: 189-200.

10. Mavroudi A, Ioannis X, Papastavrou T, Karatza E, Fotoulaki M, De Maria F, et al. Increased prevalence of Silent Celiac Disease among greek epileptic children. 
Enfermedad celíaca en epilepsia criptogénica - A. M. Madrid et al

Pediatric Neurol 2007; 36: 165-9.

11. Cronin CC, Jackson LM, Feighery C, Shanahan F, Abuzakouk M, Ryder DQ, et al. Coeliac disease and epilepsy. QJM 1998; 91: 303-8.

12. Martínez-Bermejo A, Polanco I, Royo A, López-Martin V, Arcas J, Tendero A, et al. A study of Gobbi's syndrome in Spanish population. Rev Neurol 1999; 16-31; 29: 10510.

13. Penigran D, Holmes G, Willis A. The prevalence of epilepsy in patients with celiac disease. Epilepsia 2004 45: 1291-3.

14. Ranua J, Luoma K, Auvinen A, Maki M, Haapala AM, Peltola J, et al. Celiac disease-related antibodies in an epilepsy cohort and matched reference population Epilepsy Behav 2005; 6: 388-92.
15. Mavroudi A, Karatza E, Papastavrou T, Panteliadis C, Spiroglou K. Successful treatment of epilepsy and celiac disease with a gluten-free diet. Pediatr Neurol 2005; 33: 292-5.

16. Pratesi R, Modelli IC, Martins RC, Almeida PL, Gandolfi L. Celiac disease and epilepsy: favorable outcome in a child with difficult to control seizures. Acta Neurol Scand 2003; 108: 290-3.

17. Harper E, Moses H, Lagrange A. Occult celiac disease presenting as epilepsy and MRI changes that responded to gluten-free diet. Neurology 2007; 68: 533-4.

18. Canales P, Mery VP, Larrondo FJ, Bravo FL, Godoy J. Epilepsy and celiac disease: favorable outcome with a gluten-free in a patient refractory to antiepileptic drugs Neurologist 2006; 12: 318-21. 